TUMOUR IMMUNOLOGY

\title{
Attacking the enabler
}

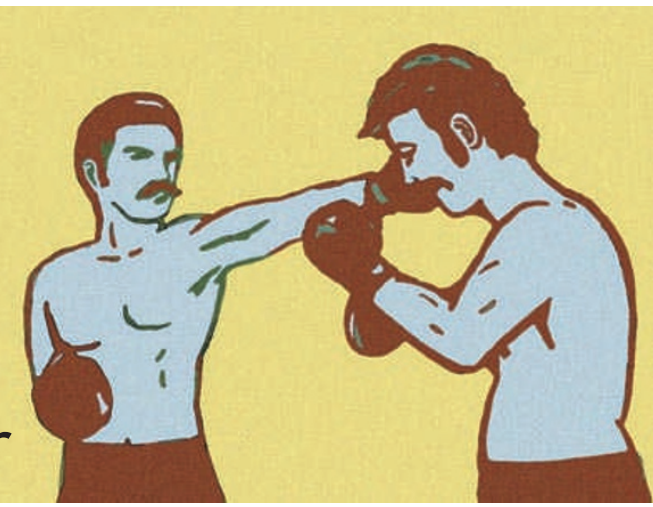

Studies of numerous experimental models of cancer have indicated that invariant natural killer T (iNKT) cells might have an antitumour role. iNKT cells recognize glycolipids presented in the context of the MHC-like molecule CD1d, but most cancer cells do not express CD1d. So how do iNKT cells recognize tumours and restrict their growth? Song et al. now show that recognition of tumour-derived antigens by iNKT cells is mediated by CD1 $\mathrm{d}^{+}$monocytes and macrophages.

After confirming that tumourassociated macrophages express CD1d and colocalize with iNKT cells in frozen neuroblastoma tumour tissue samples, the authors investigated whether monocytes could crosspresent tumour-derived antigens to iNKT cells. Adding iNKT cells to peripheral blood mononuclear cells pulsed with tumour lysates resulted in high levels of monocyte killing and interferon- $\gamma$ (IFN $\gamma$ ) secretion. This was inhibited by the addition of a CD1d-blocking antibody, suggesting a role for iNKT cells in this process. The glycolipid antigen that was presented to iNKT cells was derived from the tumour cells themselves and was not generated by the monocytes, as pre-treatment of the tumour cells, but not of monocytes, with an inhibitor of glucosylceramide synthase (the enzyme required for glycosphingolipid synthesis) decreased IFN $\gamma$ secretion.

But how does the killing of antigen-presenting monocytes restrict tumour growth? In vitro experiments showed that monocytes directly promote tumour cell growth by secreting interleukin- 6 in response to factors secreted by the tumour cells; this was confirmed by in vivo adoptive transfer experiments.

To examine whether iNKT cells can kill monocytes and thereby prevent tumour growth in vivo, the authors injected mice with a neuroblastoma cell line and monocytes and compared tumour growth with mice that had also been injected with iNKT cells. As predicted, co-injection of mice with iNKT cells prevented monocyte-mediated promotion of tumour growth; this effect was inhibited by the addition of a CD1d-blocking antibody, thereby confirming the in vitro findings. The antitumour effect exerted by iNKT cells was found to be dose dependent, with a higher ratio of iNKT cells to monocytes resulting in increased inhibition of tumour growth.

These findings suggest that monocytes and macrophages can cross-present tumour-derived glycolipid antigens to iNKT cells, which then kill monocytes and prevent them from promoting tumour growth. Further research into the details of this process could provide useful information for the design of tumour immunotherapies.

Rachel David

ORIGINAL RESEARCH PAPER Song, L. et al.

Va24-invariant NKT cells mediate antitumor activity via killing of tumor-associated macrophages.J. Clin. Invest. 1 May 2009 (doi:10.1172/JCl37869) 